\title{
Capacidade de parasitismo de Trichogramma exiguum Pinto \& Platner, 1978 (Hymenoptera: Trichogrammatidae) em ovos de Plutella xylostella (L., 1758) (Lepidoptera: Plutellidae) em diferentes temperaturas
}

\author{
Parasitism capacity of Trichogramma exiguum Pinto \& Platner, 1978 (Hymenoptera: \\ Trichogrammatidae) on different temperatures on Plutella xylostella (L., 1758) \\ (Lepidoptera: Plutellidae) eggs
}

\author{
Fabricio Fagundes Pereira $^{\mathrm{I}^{*}}$ Reginaldo Barros ${ }^{\mathrm{II}}$ Dirceu Pratissoli ${ }^{\mathrm{III}}$ \\ Cácia Leila Tigre Pereira ${ }^{\mathrm{IV}}$ Ulysses Rodrigues Vianna ${ }^{\mathrm{I}}$ \\ José Cola Zanuncio ${ }^{\mathrm{I}}$
}

RESUMO

Estudou-se a capacidade de parasitismo de Trichogramma exiguum Pinto \& Platner, 1978 (Hymenoptera: Trichogrammatidae) em ovos de Plutella xylostella (L., 1758) (Lepidoptera: Plutellidae) nas temperaturas de 18, 20, 22, 25, 28,30 e $32^{\circ} \mathrm{C}$, avaliando-se o número de ovos parasitados diariamente, a porcentagem acumulada de parasitismo, o número total de ovos parasitados por fêmea e a longevidade de fêmeas. $O$ ritmo de parasitismo, durante as primeiras 24 horas, oscilou de 1,5 a 11,7 ovos parasitados por fêmeas de $\mathbf{T}$. exiguum, nas temperaturas entre 18 e $32^{\circ} \mathrm{C}$. O parasitismo acumulado de ovos de $\boldsymbol{P}$. xylostella, nas temperaturas de 18 , $20,22,25,28,30$ e $32^{\circ} \mathrm{C}$, atingiu $80 \%$, respectivamente, aos $10,7,8,5,5,4$ e 5 dias, por T. exiguum. As maiores taxas de parasitismo obtidas por T. exiguum ocorreram nas faixas térmicas de 25, 30 e $32^{\circ} \mathrm{C}$. A longevidade de fêmeas de $\mathbf{T}$. exiguum, nas faixas térmicas compreendidas entre 18 e $32^{\circ} \mathrm{C}$, variou de 4,2 a 7,4 dias.

Palavras-chave: parasitóide, controle biológico, traça-dascrucíferas, temperatura.

\section{ABSTRACT}

The parasitism capacity of Trichogramma exiguum Pinto \& Platner, 1978 (Hymenoptera: Trichogrammatidae) on eggs of Plutella xylostella (L., 1758) (Lepidoptera: Plutellidae) was studied, under temperatures of $18,20,22,25,28,30$ and $32^{\circ} \mathrm{C}$ aiming at evaluating the number of days with parasitism, cumulated parasitism, total number of eggs parasited per female and their longevity. Parasitism during the first 24 hours ranged from 1.5 to 11.7 eggs of P. xylostella per T. exiguum female in the range of 18 to $32^{\circ} \mathrm{C}$. Cumulated egg parasitism of $\boldsymbol{P}$. xylostella by $\boldsymbol{T}$. exiguum reached $80 \%$ after $10,7,8,5,5,4$ and 5 days at 18 , $20,22,25,28,30$ and $32^{\circ} \mathrm{C}$. Higher parasitism rates were recorded at 25, 30 and $32^{\circ} \mathrm{C}$ while longevity of $\boldsymbol{T}$. exiguum females varied from 4.2 to 7.4 days under temperatures of 18 to $32^{\circ} \mathrm{C}$.

Key words: parasitoid, biological control, diamondback moth, temperature.

\section{INTRODUÇÃO}

Plutella xylostella (L., 1758) (Lepidoptera: Plutellidae) é a principal praga das crucíferas em escala mundial, sendo responsável por grandes perdas em plantios comerciais de repolho (CASTELO BRANCO \& GATEHOUSE, 2001). O controle químico é o método de supressão populacional mais utilizado para $\boldsymbol{P}$. xylostella; entretanto, alguns inseticidas recomendados para controle dessa praga vêm sofrendo restrições de uso por serem ineficientes, devido ao surgimento de populações resistentes (CASTELO BRANCO et al., 2003).

O controle biológico de $\boldsymbol{P}$. xylostella, quando bem implantado, pode ser uma alternativa frente às habituais recomendações de controle químico

\footnotetext{
IDepartamento de Biologia Animal/ Entomologia, Universidade Federal de Viçosa (UFV). Av. Ph Rolfs, 36571-000, Viçosa, MG, Brasil. E-mail: ffpereira@insecta.ufv.br. *Autor para correspondência.

IIDepartamento de Agronomia /Fitossanidade, Universidade Federal Rural de Pernambuco (UFRPE), 52171-900, Recife, PE, Brasil.

IIIDepartamento de Fitotecnia, Universidade Federal do Espírito Santo (UFES), Centro de Ciências Agrárias, Alegre, ES, Brasil.

IVDepartamento de Fitossanidade, Universidade Estadual Paulista (UNESP), Faculdade de Ciências Agrárias e Veterinárias (FCAV), 14884-900, Jaboticabal, SP, Brasil.
} 
(KRNJAJIC et al., 1997). O grande número de trabalhos mencionando o complexo de parasitóides nas diferentes regiões produtoras de crucíferas demonstra a importância desses inimigos naturais para a manutenção do nível populacional dessa praga abaixo do nível de dano econômico (MITCHEL et al., 1998).

Dentre esses agentes de controle biológico, os parasitóides de ovos do gênero Trichogramma destacam-se pela ampla distribuição geográfica, por serem altamente especializados, como também pela comprovada eficiência no controle de pragas, sobretudo aquelas pertencentes à ordem Lepidoptera (ZUCCHI \& MONTEIRO, 1997; HAJI et al., 2002).

O sucesso de programas de controle biológico com espécies de Trichogramma depende de estudos preliminares, porque estes organismos podem ser afetados por muitos fatores, tais como hospedeiro, temperatura, arquitetura e fenologia das plantas, vento e produtos químicos(GOODENOUGH\&WITZ, 1985). Atemperatura é o fator climático que mais afeta os parasitóides desse gênero, pois, dentro de determinados limites térmicos, a taxa de desenvolvimento desses insetos pode aumentar ou diminuir (PRATISSOLI et al., 2005).

Trichogramma exiguum Pinto \& Platner, 1978 (Hymenoptera: Trichogrammatidae) foi mencionada como eficiente em relação ao seu potencial de uso no controle de $\boldsymbol{P}$. xylostella na França (TABONE et al., 1999). No Brasil, à exceção de BARROS \& VENDRAMIM (1999), PRATISSOLI et al. (2004) e PEREIRA et al. (2004 a e b), são escassas as pesquisas relatando aspectos biológicos desse inimigo natural parasitando ovos de $\boldsymbol{P}$. xylostella.

O estudo da capacidade de parasitismo de T. exiguum em função da temperatura pode fornecer importantes subsídios sobre parâmetros comportamentais envolvidos no processo de parasitismo e considerados relevantes para que o potencial desse agente de controle biológico seja efetivamente alcançado em programas de manejo integrado de $\boldsymbol{P}$. xylostella. Assim, o objetivo dessa pesquisa foi obter informações sobre aspectos biológicos de $\boldsymbol{T}$. exiguum parasitando ovos de $\boldsymbol{P}$. xylostella em diferentes temperaturas.

\section{MATERIAL E MÉTODOS}

O experimento foi conduzido no Laboratório de Entomologia do Centro de Ciências Agrárias da Universidade Federal do Espírito Santo (CCA-UFES).

\section{Criação de $\boldsymbol{P}$. xylostella}

Cerca de 500 pupas de $\boldsymbol{P}$. xylostella provenientes da criação-estoque do laboratório de
Biologia de Insetos da Área de Fitossanidade da Universidade Federal Rural de Pernambuco (UFRPE) foram transferidas para o Laboratório de Entomologia do CCA-UFES, onde foi iniciada a criação da traçadas-crucíferas em folhas de couve, segundo método adotado por BARROS \& VENDRAMIM (1999).

Coleta, manutenção e multiplicação do parasitóide

T. exiguum foi coletada em plantios de tomate, no município de Muniz Freire, ES. Para a coleta dessa espécie, foram utilizadas cartelas contendo ovos de Anagasta kuehniella (Zeller, 1879) (Lepidoptera: Pyralidae) inseridas em pequenas gaiolas confeccionadas com tela do tipo mosquiteiro, grampeadas nas folhas do terço superior de plantas de tomate. Após 48 horas, as cartelas foram recolhidas e transferidas para uma sala climatizada, a fim de observar os ovos parasitados e a emergência de adultos, os quais foram enviados posteriormente ao Dr. Roberto Antônio Zucchi, do Departamento de Entomologia da Escola Superior de Agricultura “Luiz de Queiroz”, em Piracicaba, SP, para identificação.

Para a manutenção dos parasitóides, ovos de A. kuehniella, obtidos segundo técnica adaptada de PARRA(1997), foram inviabilizados e colados com goma arábica diluída a 30\% em retângulos de cartolina azul celeste $(8,0 \times 2,0 \mathrm{~cm})$. Estas cartelas foram inseridas em tubos de vidro $(8,5$ x 2,4cm) contendo adultos recémemergidos; em seguida, os tubos foram vedados com filme de PVC, para evitar a fuga dos parasitóides. Na multiplicação, foi adotado o mesmo procedimento para manutenção, alterando-se apenas o tamanho do retângulo para $10 \times 10 \mathrm{~cm}$, a fim de se evitar o superparasitismo. Desse modo, foi permitido o parasitismo por 24 horas em sala climatizada, à temperatura de $25 \pm 1^{\circ} \mathrm{C}$, umidade relativa de $70 \pm 10 \% \mathrm{e}$ fotofase de 14 horas.

Capacidade de parasitismo de T. exiguum em ovos de P. xylostella em diferentes temperaturas.

Ovos de $\boldsymbol{P}$. xylostella com 12 horas de idade foram coletados de discos de folha de couve e transferidos com auxílio de um pincel de pêlos finos umedecidos para cartelas de cartolina azul-celeste (2,5 $\mathrm{x} 0,3 \mathrm{~cm})$. Para cada temperatura em estudo $(18,20,22$, 25,28 , 30 e $32^{\circ} \mathrm{C}$ ), foram isoladas 20 fêmeas recémemergidas, em tubos de vidro (3,0 x 0,4cm), fechados com filme plástico PVC, contendo no seu interior gotículas de mel para alimentação dos adultos. Cartelas contendo 30 ovos do hospedeiro, quantidade esta ajustada em função de testes preliminares, foram diariamente introduzidas nos tubos para cada fêmea individualizada. A troca das cartelas foi realizada 
diariamente, sempre a partir das 15 horas. As cartelas com ovos parasitados no dia anterior foram transferidas para sacos plásticos de $(23 \times 4,0 \mathrm{~cm})$, fechados e mantidos em câmaras climatizadas reguladas para as temperaturas citadas anteriormente, com umidade relativa de $70 \pm 10 \%$ e fotofase de 14 horas até a emergência dos descendentes. Foram avaliados os seguintes parâmetros biológicos: ritmo de parasitismo diário; porcentagem acumulada de parasitismo; número de ovos parasitados por fêmea e longevidade das fêmeas.

Os tratamentos foram representados pelas temperaturas de $18,20,22,25,28,30$ e $32^{\circ} \mathrm{C}$. Foram utilizadas vinte repetições em delineamento inteiramente casualizado, sendo cada uma representada por uma fêmea de T. exiguum. A escolha da equação que melhor se ajustou aos dados foi baseada no fenômeno em estudo e no coeficiente de determinação $\left(r^{2}\right)$, na significância dos coeficientes de regressão $\left(\beta_{\mathrm{i}}\right)$ e da regressão pelo teste $\mathrm{F}$ em nível de $5 \%$ de probabilidade de erro.

\section{RESULTADOS E DISCUSSÃO}

O ritmo de parasitismo diário variou com a temperatura e oscilou entre 1,5 e 11,7 ovos parasitados por T. exiguum, durante as primeiras 24 horas, nas temperaturas entre 18 e $32^{\circ} \mathrm{C}$ (Figura 1). O maior número de ovos parasitados ocorreu a $25^{\circ} \mathrm{C}$, com 12,0 ovos parasitados no primeiro dia (Figura 1 ). $\mathrm{O}$ fato de o ritmo de parasitismo não ser constante em temperaturas diferenciadas têm sido averiguado por diversos autores, os quais afirmam que estas variações estão relacionadas com a linhagem, a espécie e o hospedeiro utilizados (PRATISSOLI \& PARRA, 2001; PRATISSOLI et al., 2004).

Observou-se a ocorrência da maior concentração de parasitismo nos primeiros dias (Figura 1). Resultado semelhante foi constatado por PRATISSOLI et al. (2004) para Trichogramma pretiosum Riley, 1879 (Hymenoptera: Trichogrammatidae) parasitando ovos de $\boldsymbol{P}$. xyllostella, embora o período de parasitismo de ovos desse hospedeiro tenha sido mais longo para T. pretiosum, em relação a T. exiguum, nas temperaturas de 20, 22, 28 e $30^{\circ} \mathrm{C}$. RESENDE \& CIOCIOLLA (1996) obtiveram resultados em que o período de parasitismo em ovos de Helicoverpa zea (Boddie, 1850) (Lepidoptera: Noctuidae) por Trichogramma atopovirilia Oatman \& Platner, 1983 variou de 4 a 12 dias, com maior concentração de postura nas primeiras 24 horas, nas temperaturas de $15,20,25$ e $30^{\circ} \mathrm{C}$.
As variações de parasitismo observadas nesta pesquisa, bem como as relatadas por outros autores, podem estar relacionadas à técnica de criação utilizada, do hospedeiro natural do parasitóide, a procedência da linhagem, à geração de laboratório, às condições de temperatura, à umidade relativa, ao fotoperíodo e ao hospedeiro alternativo utilizados para manutenção das criações de laboratório (NOLDUS, 1989).

O número de ovos parasitados diariamente por fêmeas de T. exiguum diminuiu em função do tempo de parasitismo nas condições térmicas estudadas, podendo-se, desta forma, inferir que o parasitismo tende a decrescer com o tempo (Figura 1). Fato similar também foi constatado em pesquisas anteriores, porém com outras espécies de Trichogramma sobre diferentes hospedeiros (RESENDE \& CIOCIOLLA, 1996; INOUE \& PARRA, 1998; PRATISSOLI et al., 2004).

O parasitismo acumulado de ovos de $\boldsymbol{P}$. xylostella, nas temperaturas de 18, 20, 22, 25, 28, 30 e $32^{\circ} \mathrm{C}$, atingiu $80 \%$ do total de ovos parasitados para cada faixa térmica, aos 10, 7, 8, 5, 5, 4 e 5 dias, por $\mathbf{T}$. exiguum, respectivamente (Figura 1). PRATISSOLI \& PARRA (2001) atribuíram como causa da variação no parasitismo o uso de diferentes espécies e/ou linhagens de Trichogramma, assim como o hospedeiro utilizado e as condições climáticas. A confiabilidade deste fato também pode ser comprovada com os resultados obtidos por MARSTON \& ERTLE (1973), que registraram o decréscimo total do parasitismo de Trichogramma minutum Riley, 1871 (Hymenoptera: Trichogrammatidae) em ovos de Sitotroga cerealella (Oliver, 1819) (Lepidoptera: Gelechiidae), após o 23ํㅡㄴ dia, com poucos ovos parasitados depois do $10^{\circ}$ dia, bem como por INOUE \& PARRA(1998), que detectaram parasitismo de $\boldsymbol{T}$. pretiosum em ovos de $\boldsymbol{S}$. cerealella até $13^{\circ}$ dia, atingindo $80 \%$ deste parasitismo por volta do 7ํ dia e SÁ \& PARRA (1994), que, ao estudarem diferentes linhagens de $T$. pretiosum sobre ovos de Ephestia kuehniella (Zeller, 1879) (Lepidoptera: Pyralidae), observaram que, de maneira geral, o parasitismo foi maior nos primeiros quatro dias de vida da fêmea.

O maior número de ovos de $\boldsymbol{P}$. xylostella parasitados por T. exiguum foi obtido nas faixas térmicas de 25 a $32^{\circ} \mathrm{C}$, sendo, portanto, $25^{\circ} \mathrm{C}$ a temperatura mais adequada para a reprodução dessa espécie (Figura 2). PRATISSOLI et al. (2004) observaram que o parasitismo máximo de $\boldsymbol{T}$. pretiosum em ovos de $\boldsymbol{P}$. xylostella ocorreu a $30^{\circ} \mathrm{C}$. Isso pode ser atribuído ao fato de $\boldsymbol{T}$. pretiosum ter sido coletada na localidade de Alegre, ES, que apresenta normais térmicas anuais mais elevadas que Muniz Freire, ES, localidade de coleta de 

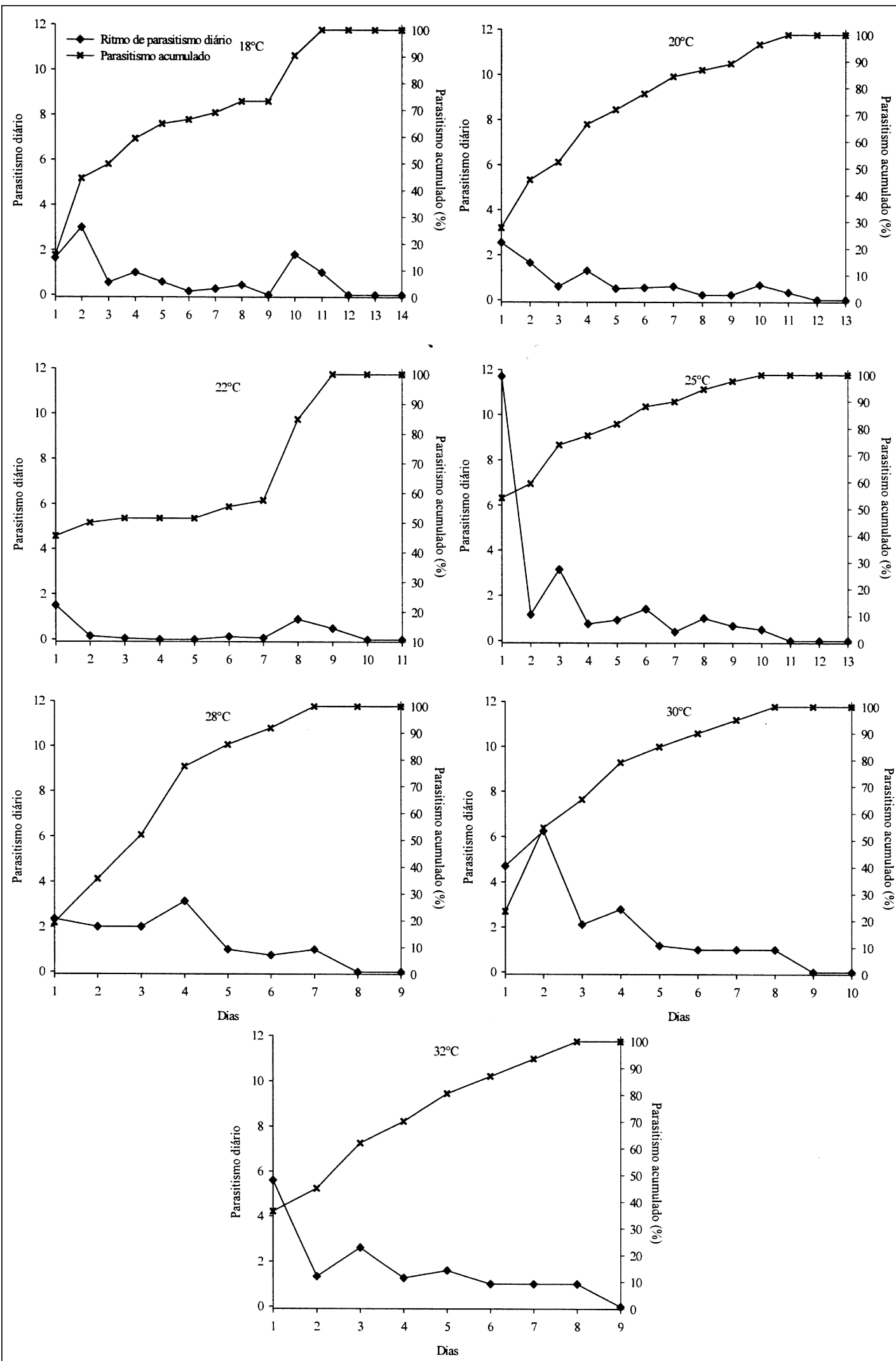

Figura 1 - Parasitismo diário e acumulado de Trichogramma exiguum sobre Plutella xylostella, sob sete temperaturas. UR: $70 \pm 10 \%$; fotofase: 14 horas.

Ciência Rural, v.37, n.2, mar-abr, 2007. 


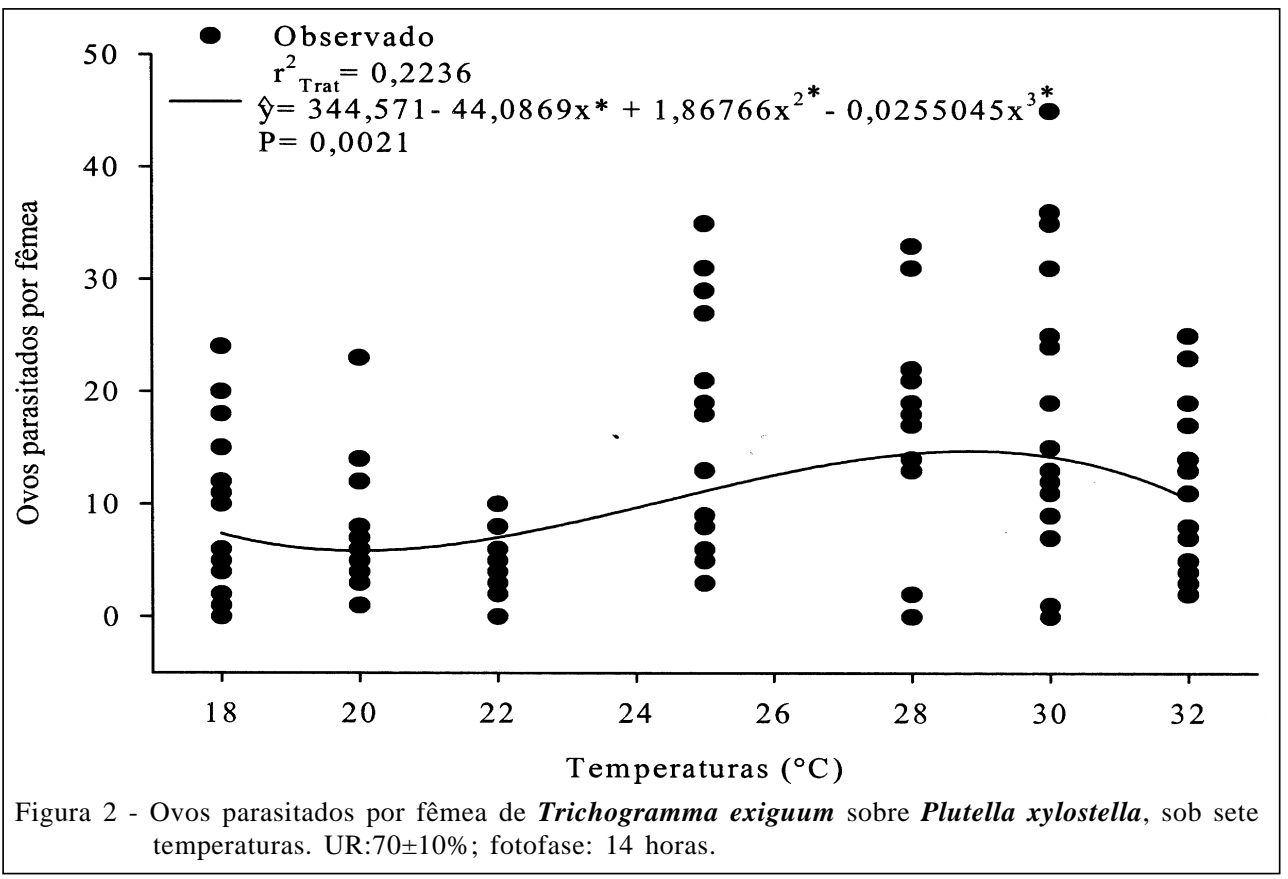

T. exiguum. Outro aspecto a se considerar, e que pode ter sido responsável pela capacidade de $\boldsymbol{T}$. pretiosum parasitar maior quantidade de ovos sob temperatura mais elevada que T. exiguum, é a habilidade de espécies do gênero Trichogramma em introduzir o ovipositor no córium do ovo hospedeiro, uma vez que este último perde gradativamente a turgidez com o aumento da temperatura.
A longevidade de fêmeas de T. exiguum em ovos de $\boldsymbol{P}$. xylostella apresentou uma relação inversa com o aumento da temperatura (Figura 3). PRATISSOLI et al. (2004) verificaram longevidades semelhantes de fêmeas de $\boldsymbol{T}$. pretiosum criadas em $\boldsymbol{P}$. xylostella nas temperaturas de 18,20 e $22^{\circ} \mathrm{C}$. A partir desta última temperatura, T. pretiosum diminuiu sua longevidade, mas mantendo-se em igualdade, exceto a $32^{\circ} \mathrm{C}$,

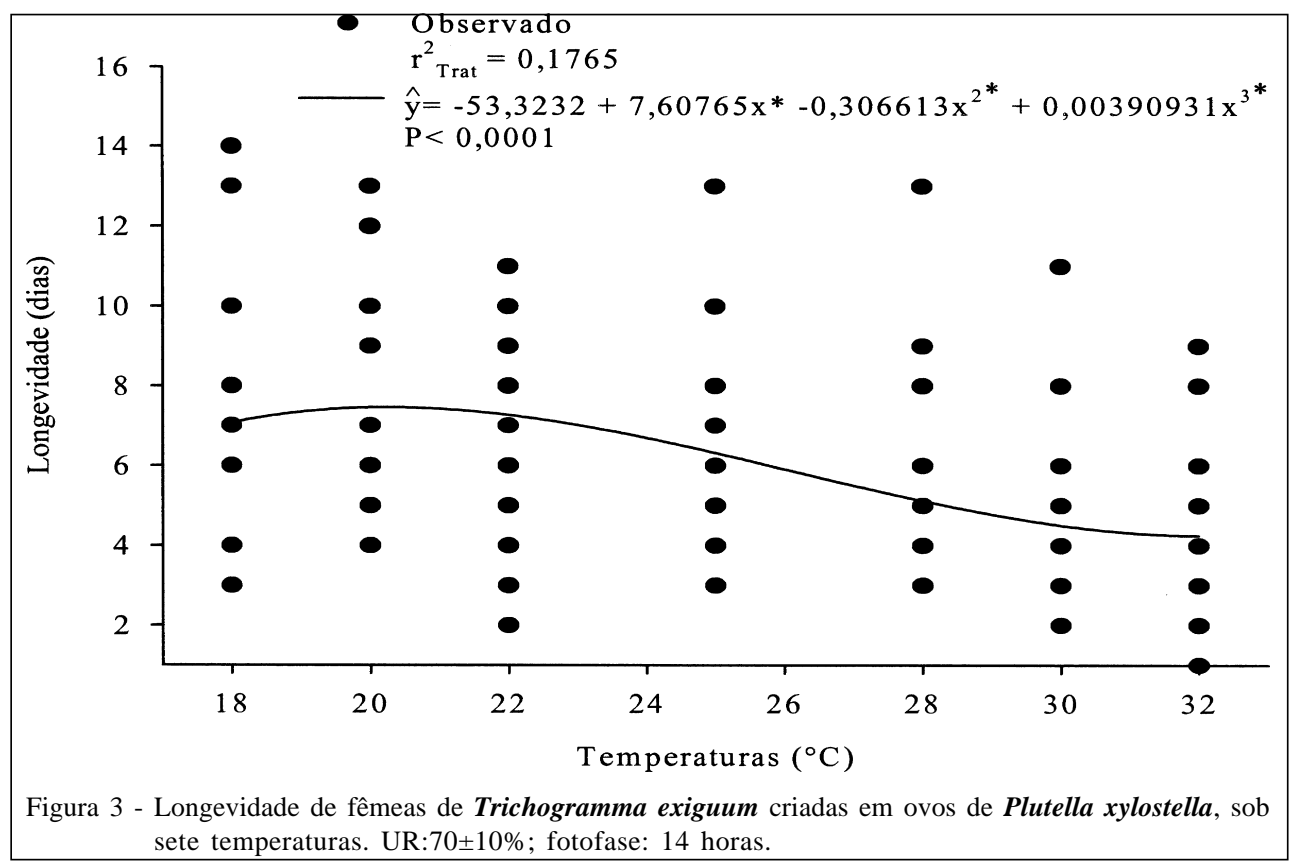

Ciência Rural, v.37, n.2, mar-abr, 2007. 
temperatura na qual se constatou o menor período de vida de suas fêmeas. De acordo com BLEICHER \& PARRA (1989), espécies diferentes, ou de locais diferentes, têm longevidades diferentes, em função da variação térmica e da alimentação. Esta reação diferenciada também foi observada por MOLINA et al. (2005) ao relatarem maior longevidade de fêmeas de $\boldsymbol{T}$. atopovirilia nas temperaturas de 20,22 e $25^{\circ} \mathrm{C}$, com valores de 8,7; 12,6 e 10 dias, respectivamente, enquanto T. pretiosum teria apresentado maior longevidade para fêmeas a 22 e $25^{\circ} \mathrm{C}$, com 9,5 e 9,1 dias, respectivamente.

\section{CONCLUSÕES}

A maior capacidade de parasitismo de $\boldsymbol{T}$. exiguum sobre ovos de $\boldsymbol{P}$. xylostella ocorre nas primeiras 24 horas de vida da fêmea desse parasitóide, independentemente da temperatura. Fêmeas de $\boldsymbol{T}$. exiguum apresentam maior longevidade reprodutiva na temperatura de $25^{\circ} \mathrm{C}$. Isso demonstra que esse agente de controle biológico poderá expressar maior eficiência no controle de $\boldsymbol{P}$. xylostella em localidades que apresentarem condições térmicas semelhantes.

\section{AGRADECIMENTOS}

À Coordenação de Aperfeiçoamento de Pessoal de Nível Superior (CAPES), pela concessão da bolsa de estudo ao primeiro autor; ao Conselho Nacional de Desenvolvimento Científico e Tecnológico (CNPq); à Universidade Federal Rural de Pernambuco (UFRPE) e ao Laboratório de Entomologia do Centro de Ciências Agrárias da Universidade Federal do Espírito Santo (CCA-UFES), por possibilitarem a execução desta pesquisa.

\section{REFERÊNCIAS}

BARROS, R.; VENDRAMIM, J.D. Efeito de cultivares de repolho, utilizados para criação de Plutella xylostella (L.) (Lepidoptera: Plutellidae), no desenvolvimento de Trichogramma pretiosum Riley (Hymenoptera: Trichogrammatidae). Anais da Sociedade Entomológica do Brasil, Londrina, v.28, n.3, p.469-476, 1999.

BLEICHER, E.; PARRA, J.R.P. Espécies de Trichogramma parasitóides de Alabama argillacea. I. Biologia de três populações. Pesquisa Agropecuária Brasileira, Brasília, v.24, n.8, p.929-940, 1989.

CASTELO BRANCO, M.; GATEHOUSE, A. Survey of insecticide susceptibility in Plutella xylostella (L.) (Lepidoptera: Yponomeutidae) in the Federal District, Brazil. Neotropical Entomololgy, Londrina, v.30, n.2, p.327-332, 2001.

CASTELO BRANCO, M. et al. Avaliação da suscetibilidade a inseticidas em populações de traça-das-crucíferas em algumas áreas do Brasil. Horticultura Brasileira, Brasília, v.21, n.3, p.549-552, 2003.
GOODENOUGH, J.L.; WITZ, J.A. Modeling augmentative releases of Trichogramma pretiosum. The Soutwestern Entomologist, Dallas, v.8, n.1, p.169-189, 1985.

HAJI, F.N.P. et al. Trichogramma pretiosum para o controle de pragas no tomateiro industrial. In: PARRA, J.R.P. et al. (Eds). Controle biológico no Brasil: parasitóides e predadores. São Paulo: Manole, 2002. Cap.28, p.477-494.

INOUE, M.S.R.; PARRA, J.R.P. Efeito da temperatura no parasitismo de Trichogramma pretiosum Riley, 1879 sobre ovos de Sitotroga cerealella (Olivier, 1819). Scientia Agricola, Piracicaba, v.55, n.2, p.222-226, 1998.

KRNJAJIC, S. et al. Biological control of cabbage pests. Acta Horticulturae, Wageningen, v.462, n.1, p.119-124, 1997.

MARSTON, N.; ERTLE, L.R. Host influence on the bionomics of Trichogramma minutum. Annals of the Entomological Society of America, Lanham, v.66, n.5, p.1155-1162, 1973.

MITCHELL, E.R. et al. Diamondback moth (Lepidoptera: Plutellidae) infestation and parasitism by Diadegma insulare (Hymenoptera: Ichneumonidae) in collards and adjacent cabbage fields. Florida Entomologist, Gainesville, v.80, n.1, p.54-61, 1998.

MOLINA, R.M.S.et al. Seleção de Trichogramma spp., para controle de Ecdytolopha aurantiana, com base na biologia e exigências térmicas. Revista Brasileira de Entomologia, Curitiba, v.49, n.1, p.152-158, 2005.

NOLDUS, L.P.J.J. Semiochemicals, foraging behaviour and quality of entomophagous insects for biological control. Journal of Applied Entomology, Hamburg, v.108, n.1, p.425451, 1989.

PARRA, J.R.P. Técnicas de criação de Anagasta kuehniella, hospedeiro alternativo para produção de Trichogramma. In: PARRA, J.R.P.; ZUCCHI, R.A. Trichogramma e o controle aplicado. Piracicaba: FEALQ, 1997. Cap.12, p.121-150.

PEREIRA, F.F. et al. Biologia e exigências térmicas de Trichogramma pretiosum Riley e T. exiguum Pinto \& Platner (Hymenoptera: Trichogrammatidae) criados em ovos de Plutella xylostella (L.) (Lepidoptera: Plutellidae). Neotropical Entomology, Londrina, v.33, n.2, p.231-236, 2004a.

PEREIRA, F.F. et al. Desempenho de Trichogramma pretiosum Riley e T. exiguum Pinto \& Platner (Hymenoptera: Trichogrammatidae) submetidos a diferentes densidades de ovos de Plutella xylostella (Lepidoptera: Plutellidae). Ciência Rural, Santa Maria, v.34, n.6, p.1669-1674, 2004b.

PRATISSOLI, D.; PARRA, J.R.P. Seleção de linhagens de Trichogramma pretiosum Riley, 1879, para o controle das traças, Tuta absoluta (Meyrick) e Phthorimaea operculella (Zeller). Neotropical Entomology, Londrina, v.30, n.2, p.277282, 2001.

PRATISSOLI, D. et al. Parasitismo de Trichogramma pretiosum em ovos da traça-das-crucíferas sob diferentes temperaturas. Horticultura Brasileira, Brasília, v.22, n.4, p.754-757, 2004.

PRATISSOLI, D. et al. Thermal requirements of Trichogramma pretiosum and T. acacioi (Hym.: Trichogrammatidae), 
parasitoids of the Avocado defoliator Nipteria panacea (Lep.: Geometridae), in eggs of two alternative hosts. Brazilian Archives of Biology and Technology, Curitiba, v.48, n.4, p.523-529, 2005.

RESENDE, D.L.M.C.; CIOCIOLLA, A.I. Capacidade de parasitismo de Trichogramma atopovirilia Oatman \& Platner, 1983 (Hymenoptera: Trichogrammatidae) em ovos de Helicoverpa zea (Boddie, 1850) (Lepidoptera: Noctuidae) em diferentes temperaturas. Ciência e Agrotecnologia, Lavras, v.20, n.4, p.421-424, 1996.

SÁ, L.A.N.; PARRA, J.R.P. Biology and parasitism of Trichogramma pretiosum Riley (Hym., Trichogrammatidae) on Ephestia kuehniella (Zeller) (Lep., Pyralidae) and Heliothis zea (Boddie) (Lep., Noctuidae) egg. Journal of Applied Entomology, Berlin, v.118, n.1, p.38-43, 1994.

TABONE, E. et al. Aptitude de 17 souches de Trichogrammes a parasiter lateigne dês cruciferes Plutella xylostella L. em laboratoire (Lep.: Yponomeutidae). Annales de la Société Entomologique de France, Paris, v.35, n.1, p. 427- 433, 1999.

ZUCCHI, R.A.; MONTEIRO, R.C. O gênero Trichogramma na América do Sul. In: PARRA, J.R.P.; ZUCCHI, R.A. Trichogramma e o controle aplicado. Piracicaba: FEALQ, 1997. Cap.1, p.41-46. 